“ (C) 2017 IEEE. Personal use of this material is permitted. Permission from IEEE must be obtained for all other uses, in any current or future media, including

reprinting/republishing this material for advertising or promotional purposes, creating new collective works, for resale or redistribution to servers or lists, or reuse of any copyrighted component of this work in other works." 


\title{
Influence of EEG tonic changes on Motor Imagery performance*
}

\author{
M. Nascimben, Y.K. Wang, A.K. Singh, J.T. King, and C.T. Lin, Fellow, IEEE
}

\begin{abstract}
In Motor Imagery literature, performance predictors are commonly divided in four categories: personal, psychological, anatomical and neurophysiological. However these predictors are limited to inter-subjects changes. To overcome this limitation and evaluate intrasubjects performance, we tried to combine two groups of these measures: psychological and neurophysiological. As neurophysiological variables tonic changes in resting EEG theta and alpha sub-bands were considered. As psychological parameter we analyzed internalized attention and its correlates in lower alpha. We found that when internalized attention doesn't decrease, Motor Imagery performance outcome can be correctly predicted by resting EEG tonic variations.
\end{abstract}

\section{INTRODUCTION}

Brain-computer interface (i.e. BCI) is an advanced technology that massively progressed over recent decade. Motor Imagery (i.e. MI) is a subcategory of BCI device systems that detects subject's motor intention and translates it in a control signal. However, MI systems suffer of user-dependent performance variations, being this an obstacle that degrades their efficiency. BCI literature describes some inter-subject predictors of MI performance outcome as mainly related to neurophysiological [1], psychological [2] and personal characteristics [3][4]. An example of neurophysiological predictor of MI outcome is electroencephalographic (i.e. EEG) frontal gamma frequency band modulation [5]. In cognitive neurophysiology, alpha sub-bands are positively correlated with various kinds of brain functions such as memory [6][7] and visual processing [8][9]. In this context, phasic and tonic resting EEG activity are an indicators of intrasubject cognitive performance. In previous works also psychological variables have been associated with MI performance prediction [10]. Ideally when mental state

* This work was supported in part by the Army Research Laboratory under Cooperative Agreement N. W911NF-10-2-0022 and in part by Ministry of Education, Taiwan, under Grant N. 105W963.

M. Nascimben is with the Brain Research Center, National Chiao Tung University, Hsinchu, 30010, Taiwan (e-mail: msnascimben@gmail.com ).

Y. K. Wang was with Brain Research Center, National Chiao Tung University, Hsinchu, 30010, Taiwan. He is now with University of Technology, Sydney, Australia (e-mail yukaiwang@cs.nctu.edu.tw )

A. K. Singh, was with Brain Research Center, National Chiao Tung University, Hsinchu, 30010, Taiwan. He is now with University of Technology, Sydney, Australia (e-mail: avinashsingh@outlook.com ).

J. T. King is with the Brain Research Center, National Chiao Tung University, Hsinchu, 30010, Taiwan (phone: 886-3-5712121; e-mail: jtchin2@gmail.com).

C. T. Lin is with University of Technology, Sydney, Australia and Brain Research Center, National Chiao Tung University, Hsinchu, 30010, Taiwan (phone: 886-3-5712121; e-mail: chintenglin@gmail.com ) . reaches a level of attention higher than a threshold level, commanding a BCI system should be easier.

Combined analysis of attentional and cognitive processes could straighten prediction of intellectual performance between subjects. For this reason we combined attentional effort with resting EEG activity to build an intra-subject MI performance predictor. We recorded resting EEG tonic activity (i.e. 'resting state prediction') and the ability to concentrate on a task through an attentional paradigm (i.e. 'internalized attention evaluation') for further analysis.

\section{A. Internalized attention evaluation}

Complex attentional functions involve multifunctional neuronal networks. We centered our observations on internalized attention, a mental state reached when attentional resources are spent by a subject focusing on task execution ignoring external disturbances. Internalized attention is also known as internal locus of control or internal meditation which directly relates with cognitive performance [11][12]. EEG correlates of internalized attention are found in lower alpha $(8-10 \mathrm{~Hz})$ over frontal cortex.

\section{B. Resting state prediction}

Previous literature reported that resting EEG alpha rhythm is positively correlated with online BCI performance [13]. Another study found that higher alpha frequencies recorded over somatosensory area during the preparatory phase right before MI have been related to performance outcome [14]. According to these observations on cognitive performance [15] we focused our analysis on resting EEG alpha sub-bands (8-10 and 10$12 \mathrm{~Hz})$ and upper theta $(6-8 \mathrm{~Hz})$.

\section{Aim of the study}

Final goal of this paper is to identify a model for predicting MI performance based on the changes of resting EEG data. The major preliminary work is to evaluate the internalized attention from frontal lower alpha during active attentional engagement together with resting EEG tonic changes. According to our hypothesis, internalized attention is a necessary step to command a BCI. Once attentional threshold is reached, it is possible to predict MI result from resting EEG using a modified version of the general cognitive rule on performance variation as exposed by W. Klimesh [16].

\section{MATERIALS AND METHODS}

BCIGEM is a videogame derived from popular visual match-three puzzle game that includes some additional subroutines like a MI game stage and an attentional tasks 
[17]. Participants in the experiment were 10 subjects all male right-handed in age range 22-27. A 32 channels Neuroscan Synamps 2 (sampling frequency $500 \mathrm{~Hz}$, extracephalic reference) device recorded EEG simultaneously with BCIGEM gameplay. Subjects performed game sessions alone in a soundproof room under control of a video surveillance system. We monitored stress levels on each candidate using a questionnaire collecting self-reported motivation, tiredness and fatigue level. We decided for this screening to avoid interferences of psychological variables. Two different datasets was acquired. First dataset (called 'pilot/baseline recording') included eyes open resting EEG and Attentional videogame task. After this 'pilot recording' same subjects trained BCIGEM videogame for two weeks before attending the second recording. Second experiment included MI stage repeated two times, resting EEG and attentional task. These latter recording forms the 'experimental/trained dataset'. In trained dataset we assume that subjects are already aware of experiment and trained to Motor Imagery and Attentional assignment. From the difference between resting EEG and active attentional stage of the two datasets we obtained the tonic variables applied for the prediction. The MI of 'experimental/trained dataset' was used as cognitive task for performance evaluation. Data were analyzed under Matlab software.

\section{A. Motor Imagery Accuracy}

In Motor Imagery, subjects were instructed to imagine a brief hand movement (near 1s) according to an arrow direction shown on screen in front of them (at 0s time, 10 trials MI right/10 trials MI left). Near 1s before arrow appearance a cross come out on screen center indicating trial start. Each trial was analyzed in $8-30 \mathrm{~Hz}$ band over 21 electrodes. The normalized raw EEG data of a single trial was represented as a matrix, where rows are the number of channels (i.e. recording electrodes) and columns are the number of samples per channel. A spatial filter was designed according to the Common Spatial Pattern algorithm and applied to the time series for the discrimination of two populations of EEG signals related to left and right motor imagery movements (two classes). The CSP algorithm increases the signal variance for one condition while minimizing the variance for the other condition. We programmed a CSP algorithm according to [18]. Than a Linear Discriminant Analysis classifier was applied to identify Motor Imagery correct condition. Finally accuracy measure was used to calculate performance on MI task.

\section{B. Resting state prediction}

Average power spectrum of resting subjects with their eyes open was calculated from the 'pilot/baseline recording' and compared with 'experimental/trained dataset'. Three minutes of free running EEG activity was acquired for each channel. Than channels were grouped by 3 to sum up cerebral areas as:

$$
\begin{aligned}
& \text { - 'Frontal' : F3, Fz, F4; } \\
& \text { - 'Fronto Central' : FC3, FCz, FC4; } \\
& \text { - 'Central' : C3,Cz, C4; } \\
& \text { - 'Centro Parietal' : CP3, CPz, CP4; }
\end{aligned}
$$

- 'Parietal' : P3, Pz, P4

After grouping an average spectrum from each region was obtained segmenting whole recording in non-overlapping windows. Five spectrums were collected as a matrix with observations as rows and then a dimensionality reduction technique (principal component analysis, PCA) on regional spectrum matrix was applied. Dimensionality reduction using PCA allowed us to obtain a single spectrum able to preserve major features of EEG power distribution.

Differences between datasets in upper theta $(6-8 \mathrm{~Hz})$, lower alpha $(8-10 \mathrm{~Hz})$ and upper alpha $(10-12 \mathrm{~Hz})$ were calculated as a tonic activity predictor of cognitive performance. The first principal component was taken in consideration because it was found being able to describe more than $68 \%$ of explained variance.

\section{Internalized attention evaluation}

During attentional tasks of BCIGEM (average of 39 epochs) subjects had to focus their attention to improve their stamina for next stage of gameplay. We instructed them to achieve a focused state for near 10 seconds to reach the goal of the game. However we found that the ability to sustain their attentional effort was better characterize in the time interval between 3 to 8 seconds of task epoch. For this reason we compared power spectral areas during this time interval in the two 'pilot/baseline' and 'experimental/trained' recording. From raw EEG data each epoch was selected from 1s prior and 10s following the onset (i.e. lasting -1 to 10 seconds). A power spectrum time series was calculated using the fast Fourier transform (FFT) from frontal channel (Fz) for each subject. Only power in $8-10 \mathrm{~Hz}$ band was considered (Table 1).

Table 1. Internalized attention power difference in lower alpha ('pilot/baseline' - 'experimental/trained' recording)

\begin{tabular}{|c|c|c|}
\hline $\begin{array}{c}\text { Internalized } \\
\text { Attention }\end{array}$ & $\begin{array}{c}\Delta \text { Area spectral power } \\
\mathbf{( 8 - 1 0 H z )}\end{array}$ & $\begin{array}{c}\text { Area incr } / \\
\text { decr }\end{array}$ \\
\hline S1 & -0.0361 & + \\
\hline S2 & 0.2148 & + \\
\hline S3 & -0.8251 & + \\
\hline S4 & -0.5767 & - \\
\hline S6 & -0.3687 & + \\
\hline S7 & 0.3669 & - \\
\hline S8 & -0.1437 & + \\
\hline S9 & 0.1816 & + \\
\hline S10 & -0.2132 & + \\
\hline S11 & -0.0866 & + \\
\hline
\end{tabular}

Spectral area variations were tested with non-parametric Wilcoxon signed rank test. Null hypothesis that distribution has median zero was rejected at the $5 \%$ significance level $(\mathrm{p}=0.0020)$. Spectral area increase in lower alpha means a negative difference between datasets while area decrease means a positive variation (third column of Table 1). 


\section{RESULTS}

\section{A. Resting state prediction}

Analysis of upper theta and upper alpha PCA power was performed following general rule on cognitive performance interpretation [16] as reported in Table 2, considering resting EEG variations as tonic variables. Even if general individual alpha frequency is subject specific, according to motor imagery characteristics the cortical processes preparing the elaboration of sensory and motor interactions are reflected by the reduction of electroencephalographic sensorimotor rhythm in $8-12 \mathrm{~Hz}$ alpha power (or event-related desynchronization, ERD) [19]. It can be interpreted as a rhythm that regulate movement-specific sensorimotor information [20], accounted mainly by high-alpha sub-band $(10-12 \mathrm{~Hz})$ rather than low-alpha sub-band $(8-10 \mathrm{~Hz})$ variations that are generally connected with tonic alertness [21]. In fact lower alpha changes as ERD in $8-10 \mathrm{~Hz}$ appears for every kind of unspecific motor behavior [22]. This is one the reasons why we split the alpha band in two sub-bands.

Table 2. Tonic changes prediction (adapted from Klimesch, Wolfgang. "EEG alpha and theta oscillations reflect cognitive and memory performance: a review and analysis." Brain research reviews 29.2 (1999)

\begin{tabular}{|c|l|l|l|l|}
\hline & \multicolumn{2}{|l|}{$\begin{array}{l}\text { Prediction: Increasing MI } \\
\text { performance }\end{array}$} & \multicolumn{2}{l|}{$\begin{array}{l}\text { Prediction: Decreasing } \\
\text { MI performance }\end{array}$} \\
\hline & $\begin{array}{l}\text { Upper } \\
\text { Theta }\end{array}$ & $\begin{array}{l}\text { Upper } \\
\text { Alpha }\end{array}$ & $\begin{array}{l}\text { Upper } \\
\text { Theta }\end{array}$ & $\begin{array}{l}\text { Upper } \\
\text { Alpha }\end{array}$ \\
\hline $\begin{array}{c}\text { Resting } \\
\text { EEG } \\
\text { spectral } \\
\text { variation }\end{array}$ & Decrease & Increase & Increase & Decrease \\
\hline
\end{tabular}

Further evidence of this subdivision is provided also by previous cognitive studies suggesting that EEG alpha rhythm is composed by two components: a lower component in $8-10 \mathrm{~Hz}$ range associated with general or global attention and an upper component in $10-13 \mathrm{~Hz}$ reflecting direct or task specific attention [23].

Table 3 Variations in resting EEG upper theta and upper alpha can predict MI performance for 7 subject according to Table 2 outcome rule (S1, S3, S4, S6, S8, S10, S11).

\begin{tabular}{|c|c|c|c|c|c|}
\hline & S1 & S2 & S3 & $\mathrm{S} 4$ & S6 \\
\hline$\Delta 6-8 H z$ & $+1.9 \%$ & $+5.5 \%$ & $+2.4 \%$ & $-4.8 \%$ & $-10.7 \%$ \\
\hline$\Delta 8-10 H z$ & $-16.8 \%$ & $+3.2 \%$ & $-1.8 \%$ & $+16.6 \%$ & $-9.8 \%$ \\
\hline$\Delta \mathbf{1 0}-12 \mathrm{~Hz}$ & $-23.5 \%$ & $-6.4 \%$ & $-8.9 \%$ & $+11.0 \%$ & $+20.1 \%$ \\
\hline$\Delta$ Accuracy & $-11.1 \%$ & $+5.5 \%$ & $-5 \%$ & $+17.2 \%$ & $+13.33 \%$ \\
\hline & S7 & S8 & S9 & S10 & S11 \\
\hline$\Delta 6-8 H z$ & $+7.2 \%$ & $-1.8 \%$ & $+1.7 \%$ & $+1.5 \%$ & $-17.8 \%$ \\
\hline$\Delta \mathbf{8 - 1 0 H z}$ & $+8.4 \%$ & $+1.2 \%$ & $+10.8 \%$ & $+1.7 \%$ & $+15.6 \%$ \\
\hline
\end{tabular}

\begin{tabular}{|l|l|l|l|l|l|}
\hline$\Delta \mathbf{1 0 - 1 2 H z}$ & $-0.5 \%$ & $+13.3 \%$ & $+6.6 \%$ & $-3.6 \%$ & $+61.3 \%$ \\
\hline AAccuracy & $+8.33 \%$ & $+1.11 \%$ & $-5.0 \%$ & $-22.2 \%$ & $+11.1 \%$ \\
\hline
\end{tabular}

Table 3 shows sub-bands power variation of resting EEG between 'pilot/baseline' and 'experimental/trained' and MI accuracy change during 'experimental/trained' session. According to performance rule of Table 2, MI performance could be predicted for 7 subjects (S1, S3, S4, S6, S8, S10, S11). Wilcoxon signed rank test returned a significant level $(\mathrm{p}<0.05)$ on both upper theta and upper alpha sub-band differences. Lower alpha was included in Table 3 even if this sub-band is not taken in consideration during this part.

\section{B. Internalized attention evaluation}

In current study, data collected from BCIGEM attentional assignment was analyzed to understand the effect of internalized attention on a cognitive task, in our case Motor Imagery. Analysis focused on power of EEG data recorded from $\mathrm{Fz}$ channel in lower alpha band (8$10 \mathrm{~Hz})$. We found that we could predict the outcome based on rule of Table 2 in those subject who showed increase of power in lower alpha band. Power increase in lower alpha band directly reflects internalization of attention [24], [25], [26]. Subjects S1, S3, S4, S6, S8, S10, S11 in Table 1 show a general increase in lower alpha power in 'experimental/trained' dataset compared to the 'pilot/baseline' dataset. This increase in lower alpha power during attentional assignment started after 3 seconds from stimulus onset and remain stable at least for next 5 seconds approximately. Those subject who have enhanced 'internal control' reflected by a power increase in lower alpha (Table 1) also follow the rule exposed in Table 2 for cognitive performance prediction. Subjects able to focus internally excluding external distractions reflected by increase in lower alpha power allow direct application of prediction rule exposed in Table 2. In these subjects Table 2 rule allows prediction of MI $\Delta$ Accuracy shown on Table 3. Subjects S2, S7 and S9 have a power decrease in lower alpha indicating a worse focusing of attention. In these subjects the prediction rule fails.

\section{CONCLUSION}

This study proposes a method to predict MI outcome derived from general cognitive guidelines. This intrasubject approach differs from previous studies mainly targeted on inter-subjects changes. Even if results support for a more extended study of this promising procedure, it suffers of some limitations. PCA methodology by one side allowed to sum up a representation of spectral activities over all cortical areas preserving relevant information but on other side could introduce a distortion on spectral proportions. This could explain a certain lack of proportionality in bands spectral variations. Maybe supervised PCA methods could be tried in future. Another 
limitation is the number of subjects but more subjects will be recruited in future experimental sessions.

Knowing in advance the performance of a subject before he acts, just evaluating his/her resting EEG activity it is a challenging effort. Neurophysiological variables like upper theta and upper alpha can reflect mental state of an individual giving a starting evaluation of intra-day BCI overall ability to command a computerized system. Some studies carried out in the past [27] stated that psychological attitude of a subject towards technology correlate with BCI good performance. But these are general findings hard to test in a user-efficient BCI design.

In our model combination of internalized attention (psychological parameter) and resting EEG (neurophysiological variable) could give an effective prediction of performance outcome. In this framework alpha power band plays a fundamental role as an inhibiting mechanism 'suppressing' external incoming stimuli and enhancing the ability to selectively concentrate on modulation of brain signals. These signals successively will be translated into BCI interpretable commands. For example alpha components could be monitored to give a warning sound or a feedback message to the user, cognizant of a possible performance decay during task execution. On the other hand our prediction model fails on the subjects who had a lack of internalized attention (S2, S7, S9). These results underline influence of attention on cognitive processes involved in MI. In the future it could be possible to monitor them jointly to solve some opened issues on user-related BCI performance inconstancy.

\section{ACKNOWLEDGMENT}

This work was supported in part by the Army Research Laboratory under Cooperative Agreement N. W911NF-10-2-0022 and in part by Ministry of Education, Taiwan, under Grant N. 105W963.

\section{REFERENCES}

[1] Grosse-Wentrup M, Schölkopf B. A review of performance variations in SMR-based brain-computer interfaces (BCIs). In: Guger C, Allison BZ, Edlinger G, editors.Brain-comput interface res. Springer Berlin Heidelberg; 2013. p. 39-51.

[2] Hammer EM, Halder S, Blankertz B, Sannelli C, Dickhaus T, Kleih S, et al. Psychological predictors of SMR-BCI performance. Biol Psychol 2012;89(January (1)):80-6.

[3] Randolph AB. Not all created equal: individual-technology fit of brain-computer interfaces. In: 2012 45th Hawaii Int Conf Syst Sci HICSS; 2012. p. 572-8

[4] Randolph AB, Jackson MM, Karmakar S. Individual characteristics and thei reffect on predicting Mu rhythm modulation. Int J Hum-Comput Interact2010;27(1):24-37.C.

[5] Grosse-Wentrup M, Schölkopf B, Hill J. Causal influence of gamma oscillations on the sensorimotor rhythm. NeuroImage 2011;56(May (2)):837-42.

[6] Vogt F, Klimesch W, Doppelmayr M. High-frequency components in the alpha band and memory performance. J Clin Neurophysiol Off Publ Am ElectroencephalogrSoc 1998;15(March (2)):167-72.
[7] Klimesch W, Vogt F, Doppelmayr M. Interindividual differences in alpha and theta power reflect memory performance. Intelligence 1999;27(4):347-62.

[8] Salenius S, Kajola M, Thompson WL, Kosslyn S, Hari R. Reactivity of magneticparieto-occipital alpha rhythm during visual imagery. Electroencephalogr ClinNeurophysiol 1995;95(December (6)):453-62.

[9] Doppelmayr MM, Klimesch W, Pachinger T, Ripper B. The functional significance ofabsolute power with respect to eventrelated desynchronization. Brain Topogr1998;11(2):133-40.

[10] Hammer, E. M., Halder, S., Blankertz, B., Sannelli, C., Dickhaus, T., Kleih, S. \& Kübler, A. (2012). Psychological predictors of SMR-BCI performance. Biological psychology, 89(1), 80-86.

[11] R. K. Johnson and R. G. Meyer, "The locus of control construct in EEG alpha rhythm feedback,” Consult. Clin. Psychol.,42, No. 6, 913 (1974).

[12] I. N. Konareva. (2012) Locus of Psychological Control and Characteristics of the EEG Frequency Components. Neurophysiology 43, 459-467.

[13] Ahn M, Cho H, Ahn S, Jun SC, High Theta. Low alpha powers may be indicative of BCI-illiteracy in motor imagery. PLoS ONE 2013b;8(November (11)):e80886.

[14] Maeder CL, Sannelli C, Haufe S, Blankertz B. Pre-stimulus sensorimotor rhythms influence brain-computer interface classification performance. IEEE Trans Neural Syst Rehabil Eng Publ IEEE Eng Med Biol Soc 2012;20(September (5)):653-62.

[15] Hanslmayr, S., Sauseng, P., Doppelmayr, M., Schabus, M., \& Klimesch, W. (2005). Increasing individual upper alpha power by neurofeedback improves cognitive performance in human subjects. Applied psychophysiology and biofeedback, 30(1), 1-10.

[16] Klimesch, W. (1999). EEG alpha and theta oscillations reflect cognitive and memory performance: a review and analysis. Brain research reviews, 29(2), 169-195.

[17] Lance, B. J., Touryan, J., Wang, Y. K., Lu, S. W., Chuang, C. H., Khooshabeh, P., ... \& McDowell, K. (2017). Towards Serious Games for Improved BCI. Handbook of Digital Games and Entertainment Technologies, 197-224.

[18] Ramoser, H., Muller-Gerking, J., \& Pfurtscheller, G. (2000). Optimal spatial filtering of single trial EEG during imagined hand movement. IEEE transactions on rehabilitation engineering, 8(4), 441-446.

[19] Pfurtscheller, Gert, and F. H. Lopes da Silva. "EEG event-related desynchronization (ERD) and event-related synchronization (ERS)." Electroencephalography: basic principles, clinical applications and related fields, 958 (1999).

[20] Babiloni, C., Del Percio, C., Arendt-Nielsen, L., Soricelli, A., Romani, G. L., Rossini, P. M., \& Capotosto, P. (2014). Cortical EEG alpha rhythms reflect task-specific somatosensory and motor interactions in humans. Clinical Neurophysiology, 125(10), 19361945.

[21] Luck, Steven J., and Emily S. Kappenman, eds. The Oxford handbook of event-related potential components. Oxford university press, 2011.

[22] Studer, B., Koeneke, S., Blum, J., \& Jancke, L. (2010). The effects of practice distribution upon the regional oscillatory activity in visuomotor learning. Behavioral Brain Function, 6, 1-10.

[23] Pfurtscheller, G., and W. Klimesch. "Event-related desynchronization during motor behavior and visual information processing." Electroencephalography and clinical neurophysiology. Supplement 42 (1991): 58-65.

[24] J. B. Rotter, Generalized expectancies for internal versus external control of reinforcement., Psychological Monographs 609 (1966).

[25] L.I. Aftanas, S.A. Golocheikine, Human anterior and frontal midline theta and lower alpha reflect emotionally positive state and internalized attention: high-resolution EEG investigation of meditation, Neuroscience Letters, Volume 310, Issue 1, 7 September 2001, Pages 57-60

[26] Foxe, J. J., \& Snyder, A. C. (2011). The role of alpha-band brain oscillations as a sensory suppression mechanism during selective attention. Frontiers in psychology, 2, 154. 
Burde W, Blankertz B. Is the locus of control of reinforcement a predictor ofbrain-computer interface performance? In: Proc 3rd int brain-computer inter-face workshop train course; 2006. p. 76-7. 